\title{
Une protéase, la calpaïne 3, est responsable d'une forme de dystrophie des ceintures
}

Les dystrophies des ceintures (LGMD, pour limb girdle muscular dystrophy) sont des myopathies transmises sur un mode autosomique dominant ou récessif, caractérisées principalement par une atrophie progressive et une faiblesse des muscles proximaux des membres. Les symptômes apparaissent durant les vingt premières années de la vie et évoluent progressivement, la perte de la marche survenant le plus souvent dix à vingt ans plus tard. La maladie s'exprime avec une grande hétérogénéité clinique. En outre, l'absence de définition nosologique spécifique rend difficile la distinction entre les malades LGMD et ceux présentant d'autres maladies neuromusculaires (telles que les dystrophies facio-scapulo-humérales [1], les myopathies de Becker, et surtout les amyotrophies spinales [2]), conditions qui ont conduit Michel Fardeau à qualifier le diagnostic LGMD de diagnostic "fourre-tout".

C'est dans ce contexte particulier qu'il faut comprendre l'importance de la découverte par Michel Fardeau et Dominique Hillaire au sud de l'Ile de La Réunion d'une importante concentration de familles comprenant une proportion élevée de malades, dont les données cliniques correspondent aux critères de la forme récessive de la LGMD, tels qu'ils furent décrits il y a plus d'un siècle par W. Erg.

C'est sur cet ensemble de familles, caractérisées par un fort taux de consanguinité, qu'un gène responsable de la forme récessive $L G M D 2 A$ (MIM n' 253600*) a pu être localisé sur le bras long du chromosome 15, grâce à la mise en évidence d'une liaison avec une sonde RFLP (restric-

\footnotetext{
* Numéro de la classification Mendelian inheritance in man.

tion fragment length polymorphism) au locus D15S25 [3]. La localisation génétique a ensuite été confirmée sur un ensemble de familles Amish [4]. Des études portant sur des familles brésiliennes [5] et métropolitaines ont montré l'hétérogénéité génétique de la forme récessive $\left(\mathrm{m} / \mathrm{s} n^{\circ} 9\right.$, vol. 8, p. 1005) puisque, pour certaines familles, le locus morbide a pu être exclu de la région du chromosome 15, un tiers des familles seulement semblant liées à ce chromosome. Depuis, une équipe anglaise a localisé un deuxième locus, LGMD2B, sur le chromosome 2 [6].

Nous avons récemment, première surprise de ce travail, exclu le locus $L G M D 2 A$ dans six familles Amish du sud de l'État de l'Indiana. Ces familles sont reliées par de multiples liens de consanguinité à celles du nord de cet État pour lesquelles l'implication du locus $L G M D 2 A$ avait été démontrée précédemment. Cette étude a également permis de révéler l'existence d'un troisième locus $L G M D$ puisque dans cette population le locus $L G M D 2 B$ ainsi que les loci impliqués dans d'autres maladies neuro-musculaires ont pu être exclus [7]. En raison de l'absence d'indices cytogénétique39s, biochimiques ou autres, l'identification du gène responsable de la forme LGMD2A a fait appel aux stratégies de clonage positionnel. Après sa localisation primaire, le gène a pu être encadré par deux marqueurs microsatellites : D15S129 dérivé du locus D15S25 et l'un des premiers microsatellites développé à Généthon D15S143, par analyse des recombinants présents dans les familles réunionnaises. Le criblage de la banque de chromosomes artificiels de levure (YAC) du CEPH avec les sondes correspondantes a permis l'isolement de clones qui ont été utilisés en hybridation in situ pour définir l'intervalle cytogénétique $L G M D 2 A$ en $15 q 15.1-q 21.1$. L'établissement de la carte physique de cette région sous la forme d'un continuum ininterrompu de YAC a été réalisé à l'aide de STS (sequencetagged sites) de différentes origines. Ce continuum comprenait 72 STS dont 25 polymorphes, ce qui correspond à une résolution génétique et physique de respectivement 140 et $400 \mathrm{~kb}$. Un minimum de 11 clones couvrent la région $15 \mathrm{q} 15.1$ à $15 \mathrm{q} 21.1$, estimée à 10 12 mégabases et $7 \mathrm{cM}$ [8].

Dans le but de réduire cet intervalle, l'analyse d'autres méioses ainsi que d'autres marqueurs de la région a été nécessaire. Seules les familles pour lesquelles l'appartenance au chromosome 15 a été certifiée par analyse génétique ont pu être étudiées. Le développement ciblé de nouveaux marqueurs de type microsatellite, produits à partir de YAC de la région, a permis la reconnaissance de nouveaux recombinants proximaux et distaux et, par conséquent, la réduction de l'intervalle $L G M D 2 A$ entre deux marqueurs distants de $1 \mathrm{cM}$ [9]. En outre, l'examen des haplotypes réunionnais a également mis en évidence, deuxième surprise, 6 haplotypes porteurs différents [9]. Cette hétérogénéité allélique est incompatible avec l'hypothèse d'un effet fondateur dans cette population consanguine, effet qui avait été présumé au début de cette étude.

Le nouvel intervalle correspond à une distance physique d'environ 3-4 Mb. La prévalence relativement faible et l'hétérogénéité génétique limitent le nombre de familles pouvant être utilisées dans l'analyse génétique, et par conséquent la restriction de cet intervalle par examen des recombinants. L'analyse de l' "homozygotie par descendance»* des familles consanguines n'a pas 
permis de mieux cerner les bornes. L'étude des déséquilibres de liaison a, cependant, suggéré un emplacement dans la moitié proximale de l'intervalle 15q15.1-15q15.3 défini par les recombinants.

Afin d'établir un catalogue des transcrits de cette région, la méthode de sélection d'ADNc a été choisie. L'hybridation sélective de banques d'ADNc musculaires avec l'ADN de YAC de l'intervalle a permis l'isolement de 3 gènes connus et de 2 séquences codantes précédemment localisés dans l'intervalle, ainsi que l'identification de 10 nouvelles séquences exprimées [10].

Considérant les manifestations cliniques de la maladie, nous avons choisi d'examiner de façon prioritaire, parmi les ADNc isolés par hybridation sélective, ceux correspondant à des messagers spécifiques du tissu musculaire. Un de ces ADNc, précédemment cloné par Sorimachi et al. [11], nous est ainsi apparu comme candidat fonctionnel, d'autant qu'il était localisé dans la région de plus fort déséquilibre de liaison [9]. Il code pour une protéase intracellulaire, la calpaïne (protéase à cystéine dépendante du calcium) spécifique du muscle squelettique.

Les calpaïnes sont des protéases non lysosomiales soupçonnées d'avoir un rôle régulateur. Elles comprennent deux isoenzymes ubiquitaires, CANP1 et CANP2 (présentes dans toutes les cellules de vertébrés examinées), deux protéines spécifiques de l'estomac et CANP3, propre au muscle squelettique. Les formes ubiquitaires sont constituées chacune d'une large sous-unité de $80 \mathrm{kDa}$ associée à la même petite sous-unité de $30 \mathrm{kDa}$, toutes codées par des gènes différents. Les larges sous-unités sont subdivisées en 4 domaines [12]. Les domaines I et III ne présentent pas d'analogie avec des protéines connues. Le domaine II est semblable aux autres protéases à cystéine avec un site actif composé d'une cystéine, d'une histidine et d'une asparagine [11]. Le domaine IV comprend 4 boucles EF, sites potentiels

\footnotetext{
* Excès d'homozygotie des marqueurs à proximilé du locus morbide chez les patients alleints d'une af

de liaison du calcium. Trois régions particulières, sans analogie connue, sont également présentes dans la protéine spécifique du muscle, NS IS1 et IS2, cette dernière contenant un motif de translocation nucléaire. Cette protéine est aussi caractérisée par une autolyse très rapide.

Après avoir isolé des cosmides correspondant à ce gène, nous en avons déterminé la séquence ainsi que l'organisation génomique. Le gène couvre $40 \mathrm{~kb}$ et est composé de 24 exons, codant pour une protéine de 821 acides aminés. CANP3 présente une forte analogie avec les autres calpaïnes, en particulier avec celles spécifiques du muscle d'autres espèces comme le rat et le porc. L'analyse en Northern blot montre un transcrit de 3,4 à $3,6 \mathrm{~kb}$ exprimé spécifiquement dans les muscles sans indication d'épissage alternatif.

L'analyse systématique de ce gène par la technique de détection électrophorétique des doubles brins hétérologues, par séquençage direct des exons ainsi que par analyse du produit de transcription illégitime $\left(m / s n^{\circ} 1\right.$, vol. $\left.6, p .55\right)$ dans les familles certifiées $L G M D 2 A$ a conduit à l'identification de 7 mutations différentes [13]. Elles comprennent 1 mutation stop, 1 d'épissage, 2 insertions-délétions et 3 faux sens. Il faut remarquer que le parti-pris de s'intéresser à des gènes spécifiques aurait pu s'avérer un mauvais choix. En effet, on sait aujourd'hui que de nombreuses affections lésant un tissu particulier sont, en fait, liées à l'altération de gènes exprimés dans tous les tissus. Le gène $S M A$, responsable de l'amyotrophie spinale récemment isolé, en est un éclatant exemple [2].

Compte tenu du taux élevé de consanguinité dans la communauté Amish, la mutation transformant l'arginine 769 en glutamine se retrouve à l'état homozygote chez tous les malades Amish du nord de l'Indiana (tout en étant absente chez les patients du sud de cet État). A l'inverse, dans la population réunionnaise, nous avons, à ce jour, identifié $5 \mathrm{mu}$ tations différentes, confirmant l'hétérogénéité allélique suggérée par l'analyse des haplotypes [9]. Ces observations sont en contradiction avec l'hypothèse d'un effet fondateur suggéré par les études généalogiques des familles de l'île de la Réunion. En effet, tous les malades appartiennent à un petit isolat génétique qui semble issu d'un ancêtre ayant immigré dans l'île vers 1670 . La présence de ces nombreuses mutations est surprenante, compte tenu de la prévalence de la maladie estimée à $10^{-5}$, incompatibilité que nous avons qualifiée de "paradoxe réunionnais".

Les hypothèses d'erreur sur la prévalence, d'un avantage sélectif ou d'une exposition à des effets mutagènes semblent peu probables. Nous pensons que le mode de transmission de la maladie, considéré jusqu'à présent comme monogénique, est en fait plus complexe et que l'expression des mutations de la calpaïne pourrait dépendre d'un fond génétique particulier (impliquant le génome mitochondrial ou nucléaire). Ce modèle de transmission di- ou même oligo-génique [13] pourrait également dépasser le cadre des dystrophies des ceintures et permettre d'expliquer les observations faites dans d'autres affections comme la maladie de Hurler $\left(\mathrm{m} / \mathrm{s} n^{\circ} 11\right.$, vol. 9 , p. 1289) ou la leucodystrophie métachromatique, ainsi que la difficulté de reproduire certains phénotypes dans les souris transgéniques $(\mathrm{m} / \mathrm{s}$ $n^{\circ} 5$, vol. 10, p. 565).

Après avoir confirmé le rôle de CANP3 dans l'étiologie de LGMD2A, nous avons étendu l'analyse à d'autres familles. Dix mutations (5 faux sens, 1 non sens et 4 décalages du cadre de lecture) dont deux déjà identifiées ont pu être mises en évidence, permettant la reconnaissance de 8 nouvelles familles LGMD2A. Il découle de l'étude rapportée dans Cell [13] que les mutations de la calpaïne sont nombreuses, sans mutation prédominante, et réparties sur l'ensemble du gène.

Le gène de la calpaïne 3 serait donc, troisième surprise, le premier gène connu impliqué dans l'étiologie d'une dystrophie ne faisant pas intervenir une protéine de structure, mais plutôt une enzyme protéolytique qui serait altérée ou absente chez les malades. Peu de progrès dans la compréhension de la fonction de cette calpaïne ont été faits depuis son 
identification en 1989 par un groupe japonais [11]. Il semble peu probable que cette enzyme ait un rôle dans la fusion des myoblastes comme il a été proposé pour les calpaïnes ubiquitaires, puisque la fusion semble ici s'effectuer normalement. Les calpaïnes ont vraisemblablement un rôle régulateur plutôt que de dégradation, mais les substrats de CANP3 n'ont toujours pas été identifiés.

Plusieurs hypothèses fonctionnelles peuvent être envisagées. Un mécanisme par "gain de fonction" est improbable car le fait que certaines mutations produisent une protéine largement tronquée et très probablement non fonctionnelle semble exclure que la mutation de cette protéase entraîne la dégradation ou la déstabilisation de composants du cytosquelette, de la matrice extracellulaire ou du complexe associé à la dystrophine. On ne peut, cependant, écarter l'hypothèse selon laquelle la calpaïne musculaire serait nécessaire à la maturation physiologique d'une protéine de régulation ou de structure du muscle. On peut aussi imaginer que cette protéase soit responsable du catabolisme de composants protéiques musculaires, l'absence d'une telle activité entraînant une accumulation toxique de ces composés et finalement la dégradation des fibres musculaires. Nous favorisons une autre hypothèse [13], celle de l'intervention de CANP3 dans la transmission du signal, avec une possible acti- vité régulatrice sur des facteurs de transcription spécifiques du muscle tels que MyoD. Des expériences de l'équipe japonaise utilisant des anticorps spécifiques montrent, en effet, une localisation nucléaire de la calpaïne musculaire [14]. De plus, les calpaïnes ubiquitaires semblent régler la transcription par clivage de cJun et de cFos et contrôleraient l'activité de NF-KB par la protéolyse de son inhibiteur IкB- $\alpha\left(m / s n^{\circ} 4\right.$, vol. 11, p. 642).

La démonstration du rôle de la calpaïne musculaire va permettre de vérifier l'appartenance des familles au groupe LGMD2A, de définir des critères nosologiques plus précis de cette myopathie - prélude à un meilleur diagnostic -, et d'étudier les relations génotypes/phénotypes dans cette maladie. Dans cette optique, il est envisagé de lancer un consortium international pour la recherche de mutations. La mise en évidence du rôle de cette protéase permet également d'envisager de nouvelles perspectives de pharmacothérapie. Différentes études sont entreprises afin de comprendre les mécanismes physio-pathologiques, l'évolutivité et la spécificité des signes cliniques de cette dystrophie des ceintures, de valider les hypothèses émises, et enfin d'identifier d'autres gènes responsables de cette myopathie. La construction d'un modèle animal par invalidation du gène $\operatorname{can} p 3$ murin par recombinaison homologue permet- tra de mieux étudier la physiopathologie de la maladie et, peut-être, de disposer d'un modèle animal pour les études à visée thérapeutique.

Isabelle Richard', Odile Broux ${ }^{1}$, Valérie Allamand', Françoise Fougerousse $^{1}$, Nuchanard Chiannilkulchai ${ }^{1}$, Nathalie Bourg', Lydie Brenguier ${ }^{1}$, Catherine Devaud', Patricia Pasturaud', Carinne Roudaut', Dominique Hillaire', Maria-Rita Passos-Bueno? Mayana Zatz ${ }^{2}$, Jay A. Tischfield ${ }^{3}$, Michel Fardeau ${ }^{4}$, Charles E. Jackson ${ }^{5}$, Daniel Cohen ${ }^{6}$ and Jacques S. Beck$\operatorname{mann}^{1,6}$

1. Généthon, 1, rue de l'internationale BP 60, 91002 Evry, France.

2. Dept. de Biologia, Inst. de Biocièncias, Univ de Sào Paulo, Sào Paulo, Brazil.

3. Dept. of Medical and Molecular Genetics, Indiana University School of Medicine, 975 West Walnut Street, Indianapolis IN 46202-5251, USA.

4. Unité de Recherches INSERM, U153, CNRS UA614, 17, rue du Fer-à-Moulin, 75005 Paris, France.

5. Henry Ford Hospital, Detroit, MI 48202, USA.

6.Fondation Jean Dausset, Centre d'Etudes du Polymonphisme Humain, 27, rue Juliette-Dodu 75010 Paris, France. 
1. Kaplan J. Deux percées sur le front des maladies neuro-musculaires : la myopathie facio-scapulo-humérale et la myopathie autosomique récessive maghrébine. médecine/sciences $1992 ; 8$ : 985-8. 2. Būrglen L, Lefebvre $S$, Reboullet $S$, Clermon O, Burlet P, Viollet L, Benichou B, Cruaud C, Millasseau P, Zeviani M, Le Paslier D, Frézal J, Cohen $\mathrm{D}$, Weissenbach J, Munnich A, Melki J. Identification et caractérisation d'un gène déterminant dans les amyotrophies spinales. médecine/ sciences $1995 ; 11$ : 149-51.

3. Beckmann JS, Richard I, Hillaire D, Broux O, Antignac C, Bois E, Cann H, Cottingham Jr RW Feingold N, Feingold J. A gene for limb-girdle muscular dystrophy maps to chromosome 15 by linkage. CR Acad Si III 1991 ; 312 : 141-8.

4. Young K, Foroud T, Williams P, Jackson CE Beckmann JS, Cohen D, Conneally PM, Tischfield J, Hodes ME. Confirmation of linkage of limb girdle muscular dystrophy, type-2, to chromosome-15. Genomics 1992 ; 13 : 1370-1.

5. Passos-Bueno MR, Richard I, Vainzof M, Fougerousse $F$, Weissenbach J, Broux $O$, Cohen $D$, Akiyama J, Marie SKN, Carvalho AA, Guilherme L, Kalil J, Tsanaclis AM, Zatz M, Beckmann JS Evidence of genetic heterogeneity in the autosomal recessive adult forms of limb-girdle muscula dystrophy following linkage analysis with $15 \mathrm{q}$ probes in Brazilian families. J Med Genet 1993 ; 30 : 385-7.

6. Bashir R, Strachan T, Keers S, Stephenson A Mahjneh I, Marconi G, Nashef L, Bushby KMD. A gene for autosomal recessive limb-girdle muscular dystrophy maps to chromosome 2p. Hum Mol Ge net $1994 ; 3: 455-7$.
7. Allamand V, Broux O, Bourg N, Richard I, Tischfield J, Hodes ME, Conneally PM, Fardeau M, Jackson CE, Beckmann JS. Genetic heterogeneity of autosomal recessive limb-girdle muscular dystrophy in a genetic isolate (Amish) and evidence for a new locus. Hum Mol Genet 1995 ; 4 : 459-64. 8. Fougerousse F, Broux O, Richard I, Allamand V, Pereira de Souza A, Bourg N, Brenguier L, Devaud $C$, Pasturaud $P$, Roudaut $C$, Chiannilkulcha N, Hillaire D, Bui H, Chumakov I, Weissenbach J, Cherif D, Cohen D, Beckmann JS. Mapping of a chromosome 15 region involved in limb-girdle muscular dystrophy. Hum Mol Genet $1994 ; 3$ : 285 93.

9. Allamand V, Broux O, Richard I, Fougerousse F, Chiannilkulchai N, Bourg N, Brenguier L, Devaud $C$, Pasturaud $P$, Pereira de Souza A, Roudaut C, Tischfield JA, Conneally PM, Fardeau M, Cohen $\mathrm{D}$, Jackson CE, Beckmann JS. Preferential localization of the limb-girdle muscular dystrophy type 2A gene in the proximal part of a lcM 15q15.1-q15.3 interval. Am J Hum Genet 1995 (sous presse).

10. Chiannilkulchai N, Pasturaud P, Richard 1, Auffray C, Beckmann J S. A primary expression map of chromosome 15 q15 region containing the recessive form of limb-girdle muscular dystrophy (LGMD2A) gene. Hum Mol Genet 1995 ; 4 (sous presse).

1I. Sorimachi H, Imajoh-Ohmi S, Emori Y, Kawasaki H, Ohno S, Minami Y, Suzuki K. Molecular cloning of a novel mammalian calcium-dependant protease distinct from both $\mathrm{m}$ - and mu-type Specific expression of the mRNA in skeletal muscle. J Biol Chem 1989 ; 264 : 20106-11.
12. Ohno S, Emory Y, Imajoh S, Kawasaki H, Kisargi M, Suzuki K. Evolutionary origin of a calcium dependent protease by fusing of genes for a thiol protease and a calcium-binding protein ? $\mathrm{Na}$ ture $1984 ; 312: 566-70$.

13. Richard I, Broux O, Allamand V, Fougerousse F, Chiannilkulchai N, Bourg N, Brenguier L, Devaud $C$, Pasturaud $P$, Roudaut $C$, Hillaire D, Passos-Bueno MR, Zatz M, Tishfield JA, Fardeau M, Cohen D, Jackson CE, Beckmann JS. Mutations in the proteolytic enzyme, calpain 3, cause limb-girdle muscular dystrophy type 2A. Cell $1995 ; 81$ : (sous presse).

14. Sorimachi H, Toyama-Sorimachi N, Saido TC, Kawasaki H, Sugita H, Miyasaka M, Arahata K, Ishiura S, Suzuki K. Muscle-specific calpain, p94, is degraded by autolysis immediately after translation, resulting in disappearance from muscle. $J$ Biol Chem 1993 ; 268 : 10593-605.

\section{Remerciements}

Nous remercions l'apport de C. Auffray, B. Bakker, P. Bucher, I. Cadjee,. H. Cann, D. Caterina, P.M. Conneally, N. Fonknechten, D. Fugman, et J. Weissenbach. Mais avant tout, nous remercions tout particulièrement les patients, leurs familles et cliniciens et Bernard Barataud sans la participation et les encouragements duquel ce travail n'aurait jamais vu le jour. Ces recherches ont bénéficié du soutien de l'Association Française contre les Myopathies. 